\title{
Virtual Mannequin Simulation for Customized Electromagnetic Shielding Maternity Garment Manufacturing
}

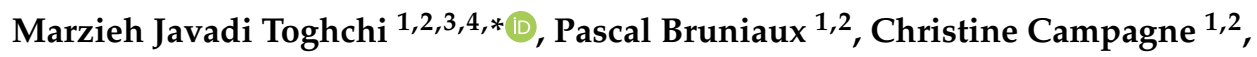 \\ Aurélie Cayla ${ }^{1,2} \mathbb{1}$, Carmen Loghin ${ }^{3}$, Irina Cristian ${ }^{3}$, Yan Chen ${ }^{4}(\mathbb{D}$ and Lichuan Wang 4 (1) \\ 1 École Nationale Supérieure des Arts et Industries Textiles, GEMTEX, 59056 Roubaix, France; \\ pascal.bruniaux@ensait.fr (P.B.); christine.campagne@ensait.fr (C.C.); aurelie.cayla@ensait.fr (A.C.) \\ 2 University of Lille, Nord de France, 59000 Lille, France \\ 3 Faculty of Textile, Leather and Industrial Management, Gheorghe Asachi Technical University, 700050 Iasi, \\ Romania; cloghin@tex.tuiasi.ro (C.L.); irina.cristian@tex.tuiasi.ro (I.C.) \\ 4 College of Textile and Clothing Engineering, Soochow University, Suzhou 215021, China; \\ yanchen@suda.edu.cn (Y.C.); lcwang@suda.edu.cn (L.W.) \\ * Correspondence: marzieh.javadi-togh@ensait.fr
}

Received: 13 November 2019; Accepted: 3 December 2019; Published: 9 December 2019

\begin{abstract}
Trying virtual garments on a virtual body has become widespread in the design industry as a result of 3D computer-aided design (CAD) system and progress in body measurement systems. Spending less time and cost compared to traditional methods of garment design is the main benefit of design software development. Moreover, it leads to improved garment fitting on the body which fulfills customer desires. In the present study, a parametric graphical method was utilized to develop a 3D virtual mannequin for the pregnancy period. The former, the 3D adaptive mannequin model was predicted based on a woman's weight by analyzing body morphology evolution of a pregnant woman. The mannequin was verified by the results of another pregnant body. Then, the developed mannequin based on weight gain during maternity was applied to design a garment block pattern. The virtual try-on of the developed garment block pattern suggested that it was well-fitted on the 3D virtual mannequin while two armpit darts and two elastic seams were allocated. Hence, the developed garment block pattern will be used to make a personalized protective garment in our future work using an electromagnetic shielding woven fabric formerly manufactured by our group.
\end{abstract}

Keywords: pregnant body morphology; 3D virtual mannequin; protective block pattern; weight gain during pregnancy

\section{Introduction}

Pregnancy mannequins are commercially available and manufactured by different companies. Although these mannequins are practical in pregnancy garment design, they are unable to show body evolution during pregnancy. Also, these mannequins are not applicable to every individual pregnant body due to the fact that they are manufactured based on standard sizes. Some companies have added the options of changing the height or abdomen line of the mannequin but these options are also limited. Thus, there is no connection between the available maternity mannequins in stock and the real morphology of pregnant women. In fact, there are not sufficient mannequins of pregnant women to help the designer to design fitted garments for the pregnancy period. Therefore, it is essential to make an appropriate mannequin for the maternity period in order to design a well-fitted garment for a pregnant woman. The designed garment will be used to produce a comfortable electromagnetic shielding garment for a pregnant woman in our future work. The protective garment has to be 
well-fitted on the pregnant body in order to attain desired protection against radiation for both mother and fetus.

Therefore, in the present study, a 3D virtual mannequin model for pregnant women has been developed. Also, the developed 3D virtual mannequin has been applied to design a garment block pattern for pregnant women that will be used to generate a real personalized electromagnetic shielding pregnancy garment in our further work. It should be taken into consideration that the garment block pattern was designed using a shielding fabric formerly manufactured by our group while the shielding level was reasonable for personal protection against electromagnetic waves (shielding effectiveness $\geq 10 \mathrm{~dB}$ ) [1].

\section{Literature Review}

Anthropometry information was applied in the design process to declare ergonomics and provide appropriate products for consumers [2]. The apparel and clothing industry has been traditionally using two-dimensional patterns to make sample garments that are fitted on a 3D dimensional mannequin. This process is generally time-consuming and ineffective [3,4]. In recent years, 3D design software has led to significant improvement in garment design by overcoming the limitations of the traditional $2 \mathrm{D}$ design methods [5-11]. The main idea was to launch a new concept of garment making to optimize the time of design, final cost, comfort and fit.

Body morphology data is a fundamental key to design a fitted garment adapted to the human body. For this reason, for example, a virtual mannequin of the human body was created based on body morphology [5]. The body was divided into three main planes in their work and the segments of torso, legs, and arms were modeled to make a 3D symmetrical adaptive mannequin. This mannequin was able to be adjusted according to the beauty and subject height. Their model was appropriate to apply in the ready-to-wear garment sectors.

Moreover, parametric mannequins have been examined based on mathematical interpolation functions of input dimensions [12-15]. For instance, anatomical feature points of a human body were extracted and the skeleton model was made based on those feature points [12]. Then, a parametric wireframe with a controllable structure was created and the human model was estimated with a sweep surface. Their proposed method was found to make human deformation really convenient for obtaining the skeleton and parametric structure. Also, a framework for creating feature-based human bodies according to the specified measurement dimensions was presented [13]. Since the parametric design algorithm of his work was example-based, the exact dimensions given cannot be achieved using this proposed method and the input of the human structure was limited to the sizing dimensions.

In addition, sizing systems used by apparel companies have been updated considering the variety of human morphologies. For instance, an intelligent system for virtual try-on was defined by body-scanning data [16]. To that end, the geodesic distribution based on anthropometrics feature points of the human torso was considered as inputs for the clustering methods. Hence, a geometrical model linked with reverse engineering techniques was applied to devise the $3 \mathrm{D}$ virtual parametric mannequins from the 3D body scanning.

Furthermore, the body morphology analysis of disabled people has been also considered by some researchers in order to design a fashioned and comfortable garment for this group of people [17-19]. For instance, a method was introduced to create two-dimensional (2D) block patterns for physically disabled people with scoliosis using three-dimensional (3D) virtual technology [17]. In another study, the prototyping of garments was generated for paraplegics in a sitting position using the body scan data [18]. The pants and blouse basic pattern designs for a standing position were constructed for the tested person by using a construction system. They concluded that the virtual prototype of the garment is practical in garment design for paraplegics. Moreover, 3D body scanning has been suggested by a number of studies for external measurement of the body parts for clinical practice in order to avoid the patient being exposed to radiation $[20,21]$. 
Ease allowance is also one of the important features discussed in a few publications. For example, a model of ease allowance for garments based on a 3D reverse procedure was introduced [22]. The body morphology analysis made it possible for a garment to be virtually designed. They concluded that the results were useful to design a customized garment on a virtual body for the ready-to-wear sector.

Also, the morphology data has been applied in garment design. For instance, a new set of variables was defined regarding stature, crotch and leg length of body measurements and the evaluated parameters for each joint in the body skeleton [23]. The database was created based on the measurements to apply in shopping via the internet. The body was deformed according to the measurements and the garments were fitted on the deformed body for tight, loose and freely float clothes around the body. Similarly, a method for dressing virtual humans using computer-aided design (CAD) cloth data was introduced [24]. The body was simulated based on physical geometric. The geometric pre-positioning procedure placed several pieces of clothing automatically around the human body. It was said that the final fitting can be undertaken efficiently by a physically-based cloth simulation.

In addition, all research works confirm that there is an evident change in the physical appearance of a woman's body during the maternity period. According to the fact that a woman's body undergoes significant changes during nine months of pregnancy, characterization of these changes has been analyzed by a few publications [25-27]. New clothing design for women during pregnancy was proposed [28]. Clothing patterns were prepared in accordance with physical comfort for a pregnant woman. It was said that four types of changes i.e., shape, dimensions, weight, and body posture have an influence on the pattern design. A connection was established between the woman's size before pregnancy and during the last month of pregnancy. The limitation is that body evolution was not taken into account during the pregnancy period since the focus was just on the last month of pregnancy. In another study, the critical design aspects of maternity support garments were reviewed [29]. It was believed that the improvement of pregnancy garments for the modern plus-size patterns should be a response to the growing obesity as well as beauty standards.

Furthermore, one study was conducted by Sohn and Bye to create an adaptive model based on real data for the pregnancy period [30]. They have made a virtual mannequin based on body scan data to apply in garment design. They tried to virtually fit a pattern on a pregnant body considering that good fit requires a proportional balance between body and garment pattern. They suggested that the patterns changed with respect to the body changes during pregnancy although they have not increased consistently. The main drawback of their work was that the sleeveless sheath dress was not closely fitted to the body in the abdomen to the hip area. Furthermore, the lower body shape of late pregnant women in the Shanghai area of China was investigated [31]. The data of different pregnant women have been collected and analyzed. The pregnant women with different body changes were categorized and it was concluded that this data would be practical for the pattern design of maternity dresses and industries involved.

A comfortable garment had to be designed for the maternity period with the potential of shielding electromagnetic radiation as this was the ultimate goal of our research. It is noted that the analysis of body morphology during pregnancy is a critical key for garment design since the body experiences enormous changes during pregnancy. Therefore, a new model using weight changes during pregnancy was introduced given that there are not satisfactory mannequins of pregnant women to apply in the apparel and garment design industry. The former, a virtual adaptive mannequin had to be developed for the pregnancy period. The latter, the proposed method for mannequin prediction, had to be verified for the whole pregnancy length of all pregnant women. This mannequin was applied to virtually design a well-fitted garment block pattern for a pregnant mannequin. This designed garment will be applied to make an electromagnetic shield garment for a pregnant woman in our future work. The designed shield garment has to be fitted on the pregnant body for different trimesters of pregnancy in order to achieve desired protection against electromagnetic waves for both mother and fetus during pregnancy.

In should be mentioned that the synergy of carbon fillers (multiwall carbon nanotube and carbon black) inside the polymer matrix $(\mathrm{PA} 6,6)$ has been applied in our previous study to develop a new 
hybrid conductive yarn for wearable shielding application. The produced yarn was used to manufacture a compact two-ply woven fabric with hybrid conductive yarn as stuffer wefts while the shielding effectiveness of the manufactured fabric was reasonable (shielding effectiveness $\geq 10$ [dB]) $[1,32]$. Therefore, the designed garment block pattern will be applied to make a real personalized pregnancy garment using the manufactured compact two-ply woven fabric with hybrid conductive yarn as stuffer wefts in our further work.

\section{Materials and Methods}

A general methodology framework of a garment block pattern generating for pregnant women was proposed in the present study which is depicted in Figure 1.

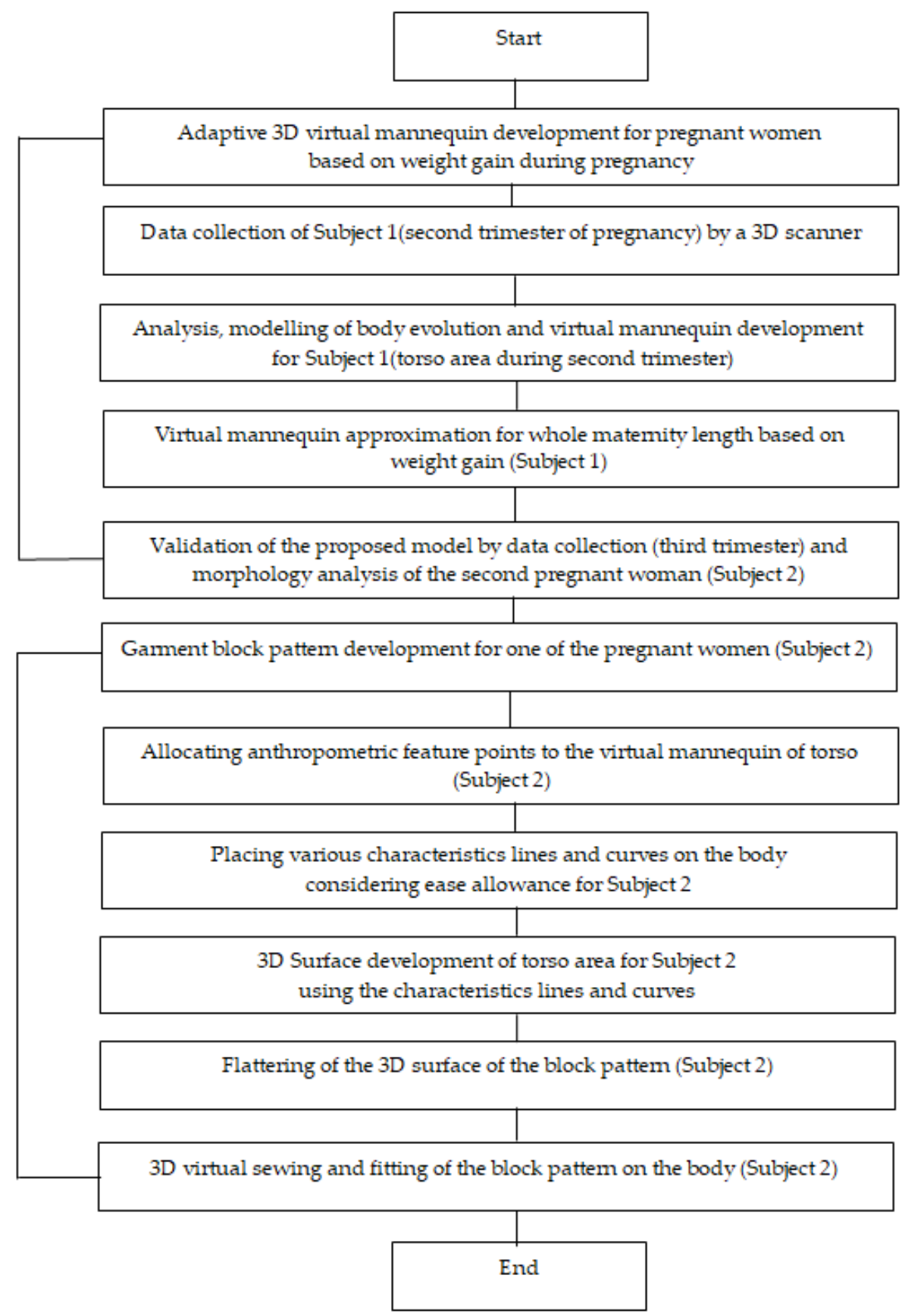

Figure 1. The general schemes of garment block generating in the present study. 


\subsection{Three-Dimensional (3D) Virtual Mannequin for Pregnant Women}

\subsubsection{Data Collection}

The pregnancy period is divided into three trimesters which each one includes three months of pregnancy period. The data for all three trimesters are listed in Table 1.

Table 1. Trimester chart.

\begin{tabular}{ccc}
\hline Trimester & Month & Week \\
\hline \multirow{2}{*}{1} & 1 & $1-4$ \\
& 2 & $5-8$ \\
& 3 & $9-13$ \\
\hline \multirow{2}{*}{2} & 4 & $14-17$ \\
& 5 & $18-21$ \\
& 6 & $22-26$ \\
\hline \multirow{2}{*}{3} & 7 & $27-30$ \\
& 8 & $31-35$ \\
& 9 & $36-40$ \\
\hline
\end{tabular}

Therefore, the body morphology of a pregnant woman (subject 1) was investigated week by week to have a comprehensive understanding of body evolution throughout pregnancy. The body has been scanned between the 16th and 26th weeks of pregnancy length. The pregnant woman was scanned using 3D Scan Tecmath supplied by Human Solution Company while she was dressed only in underwear. The subject has been asked to stand in a predefined position in the area of the accessibility of the lasers for scanning. After that, scanner data were exported to Scanwor ${ }^{\mathrm{TM}}$ software supplied by Human Solution and digitized by this software.

Then, the changes in body morphology were analyzed for the second trimester. It is noted that the body scan data of this pregnant woman was accessible for this specific duration.

It should be considered that this work concentrated on torso area as the electromagnetic shield will be applied around this area to protect both mother and fetus against the detrimental effects of electromagnetic waves [33-35].

After that, the data acquired through the body scanner had to be transformed in order to create a surface of the body that can be used in most CAD systems. For this purpose, the scanned body data of each week was first imported into Geomagic Design X software to improve the body surface and reconstruct the modified parts meanwhile scanning. Then, final surfaces were created and exported into the CAD system fit (3D Design Concept) and were used for morphology analysis. It should be noted that the data attained in Scanwor $X^{\mathrm{TM}}$ were introduced as triangular meshes to Geomagic Design X software.

\subsubsection{Analysis and Modeling of Body Evolution}

All body surfaces had to be imported into the 3D Design Concept software environment to be analyzed. Hence, the surfaces of the second trimester (16th to 26th weeks of pregnancy period) were separately transformed into 3D Design Concept and the surfaces were cleaned. Then, all the shapes were placed in a joint position so as to investigate body evolution. The bodies should be in the joint position to gain a correct understanding of body evolution. So, the body of 16th weeks of pregnancy was taken as a reference body and the legs of the scanned body for all the weeks were placed in the same position. The initial position and new position of the second scanned body on the reference body are depicted in Figure 2a,b. Although the position of legs seemed fine for the second body as given in Figure $2 b$, the spine line position was not adequate as shown in Figure $2 c, d$. That can be attributed to the fact that woman position kept on changing for each scan compared to the previous 
one. So, the spine lines had to be adjusted for all weeks according to the reference body to have the same position for all the body shapes.

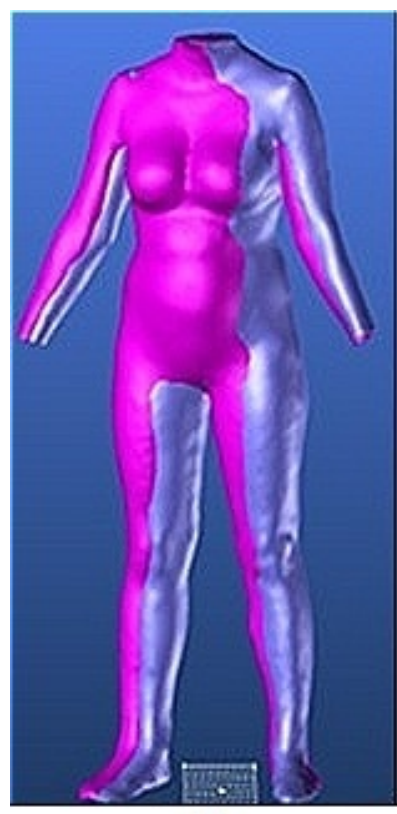

(a)

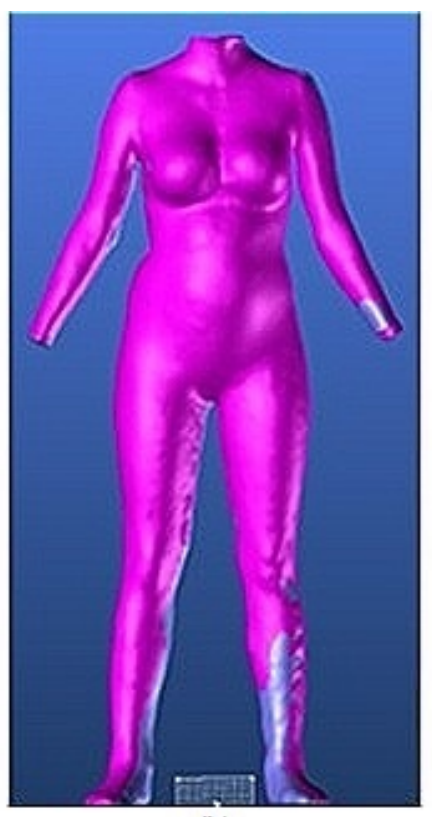

(b)

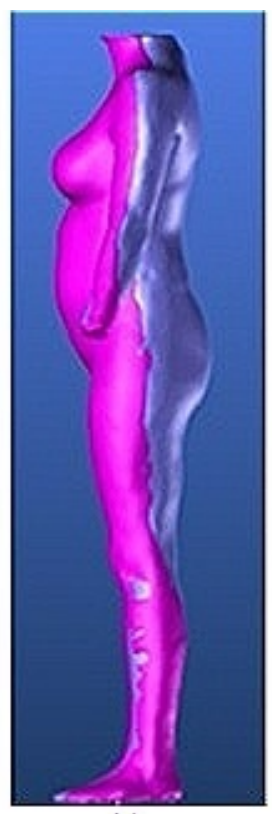

(c)

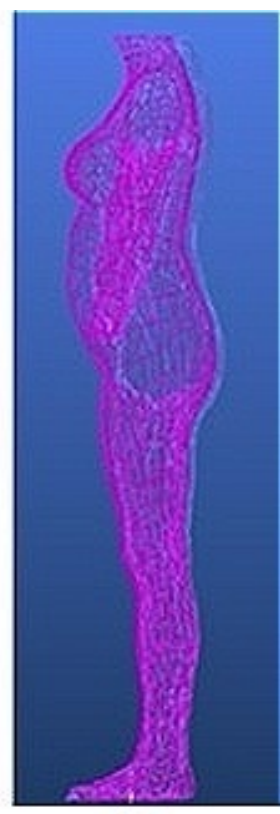

(d)

Figure 2. (a) The initial position of the pregnant body in 16th (magenta color) and 20th (silver color) weeks of pregnancy, (b) the body position based on the appropriate position of the legs, (c) and (d) the position of spine lines (Subject 1 ).

Afterward, each body was symmetrically divided into left and right parts through the spine line and all the spine lines were extracted to be compared. The spine lines positions were modified according to the reference scanned body (16th week) to facilitate the analysis of body evolution. All the spine lines positions have been adjusted considering the points under the bust, belly and waist. These three points were chosen for spine adjustment due to the observable form of the body around them. The spine lines position is depicted before and after adjustment in Figure 3a,b. As illustrated in Figure $3 b$, the evolution of the body is clear after the spinal adjustment.

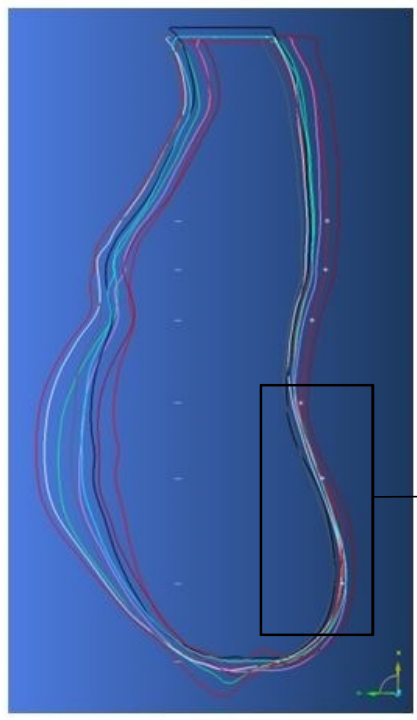

(a)

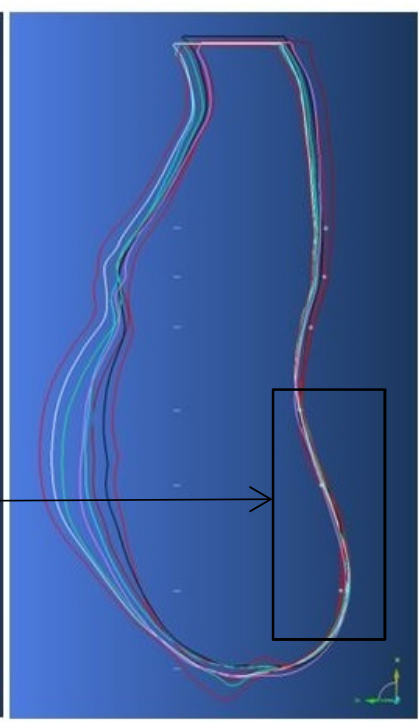

(b)

Figure 3. All the spine lines (a) before and (b) after spine position correction (Subject 1). 
Moreover, the formula of each cut is represented in Table 2. The curves were plotted for all the weeks. So, each body has been cut according to its own spine line. It should be noted that this study has focused on torso area since the final application is to protect both mother and fetus against the detrimental effects of electromagnetic waves.

Table 2. Cutting parameters.

\begin{tabular}{cc}
\hline Parameter & Formula \\
\hline Height $(\mathrm{H})$ & 1649 \\
Armpit & $0.751 \times \mathrm{H}$ \\
Breast & $0.7186 \times \mathrm{H}$ \\
Underbust & $0.685 \times \mathrm{H}$ \\
Waist & $0.63 \times \mathrm{H}$ \\
Belly & $0.58 \times \mathrm{H}$ \\
Hip & $0.51 \times \mathrm{H}$ \\
Crotch & $0.458 \times \mathrm{H}$ \\
\hline
\end{tabular}

All the curves were extracted to analyze the body evolution from 16th to 26th weeks of pregnancy. Also, these curves are necessary to define the image of clothing in the present work. The perspective view of curves is presented in Figure 4.

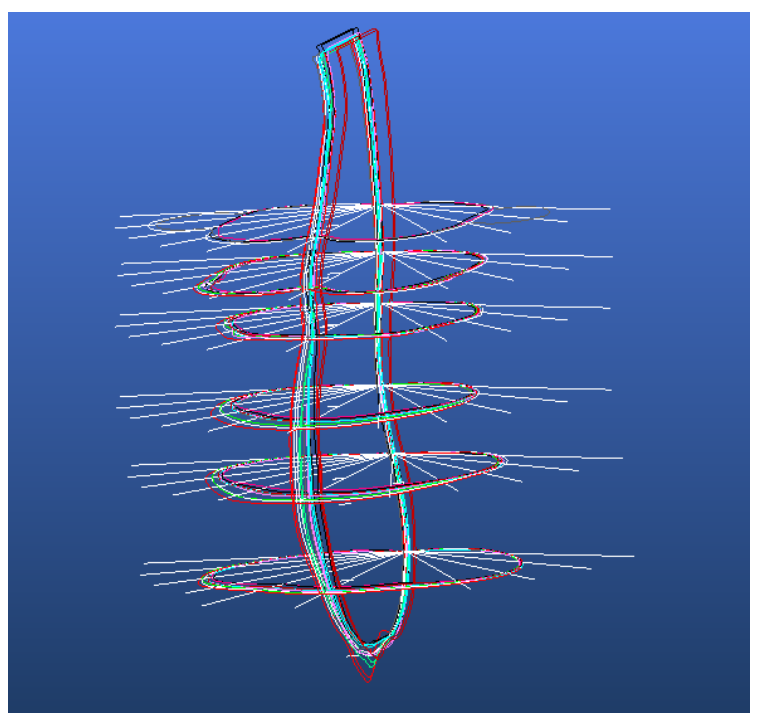

Figure 4. Curves for the 16-26th weeks of pregnancy (Subject 1).

The evolution of each curve was measured at different angles with constant intervals $\left(15^{\circ}\right)$ to have a comprehensive understanding of body evolution. The volume values from 16th to 26th weeks of pregnancy period are presented in Figure 5 . The increasing volume is generally linear while the rising rate for the belly and waist was the most among all the cuts.

Then, the growth of curves for all the cuts was measured at different angles according to the spine line as a reference. All data were sorted into an Excel file and the total rise was calculated between 16th and 26th weeks of maternity period for all the curves (between crotch and armpit). The collected data was applied to make an adaptive surface of torso using the linear upward from 16th to 26th weeks of pregnancy period. 


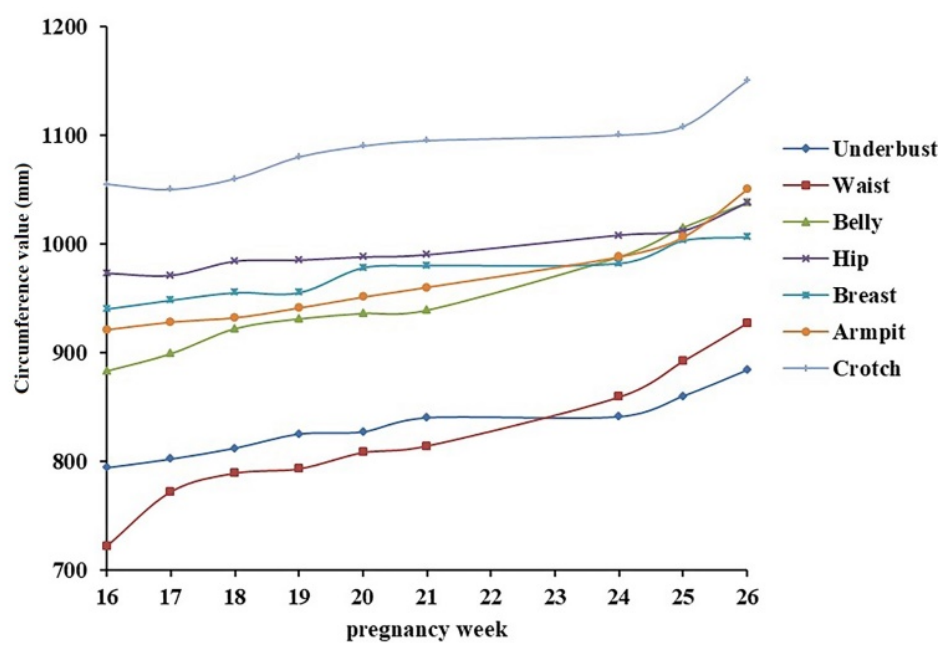

Figure 5. Circumference growth of different cross-sections in the torso for the first pregnant woman (Subject 1) between the 16th and 26th weeks of pregnancy.

\subsubsection{Virtual Mannequin Estimation for Whole Maternity Length based on Weight Gain}

It should be noted that the virtual mannequin was verified by real data obtained by the body scanner in the previous section. However, it is required to develop the virtual mannequin for the whole maternity period.

The idea was to make a virtual mannequin for weeks before 16th and after 26th weeks based on weight gain during pregnancy. Thus, the relationship between weight gain and body evolution had to be described. For this purpose, the graph of weight for the pregnant woman was characterized throughout pregnancy. The weight of this woman was $58 \mathrm{~kg}$ and $73 \mathrm{~kg}$ before pregnancy and on delivery day, respectively. It is noted that the maximum changes of body transpire in torso during pregnancy [36]. Thus, applying the correlation between weight gain and torso evolution instead of whole-body evolution remained reasonable.

Weight gain for the pregnancy period was discussed in [26,27,37-39]. The weight gain graph for the scanned woman is shown in Figure 6. Afterward, the evolution of each curve for torso was estimated based on weight gain values and the aforementioned curves of the second trimester. For this aim, the slopes of the weight curve were measured for the first and third trimester of pregnancy and the ratio between these different intervals was calculated. Then, the body evolution for these periods was predicted and the surface of torso was created for the entire maternity period. However, the results were not promising for both the first and third trimester since it did not show normal deformation for the torso and it was not coherent to the actual changes of the pregnant body.

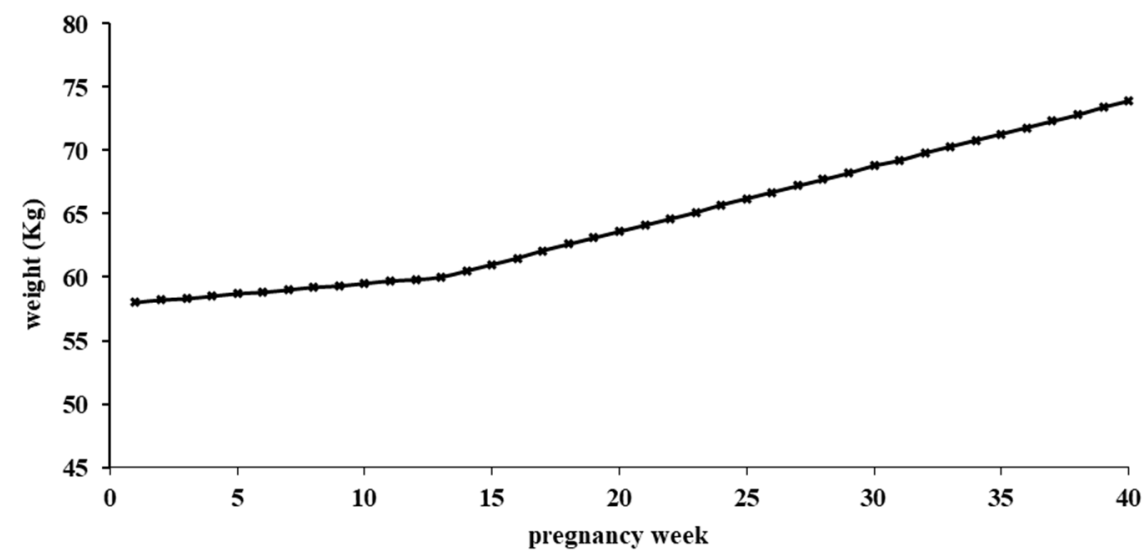

Figure 6. The weight gain graph (1) of the scanned pregnant woman (Subject 1). 
To tackle the problem of virtual mannequin prediction associated with the discussed weight gain graph, another proposed graph for weight gain during pregnancy (graph 2) was applied [27,40]. This graph for subject 1 is depicted in Figure 7 (solid line).

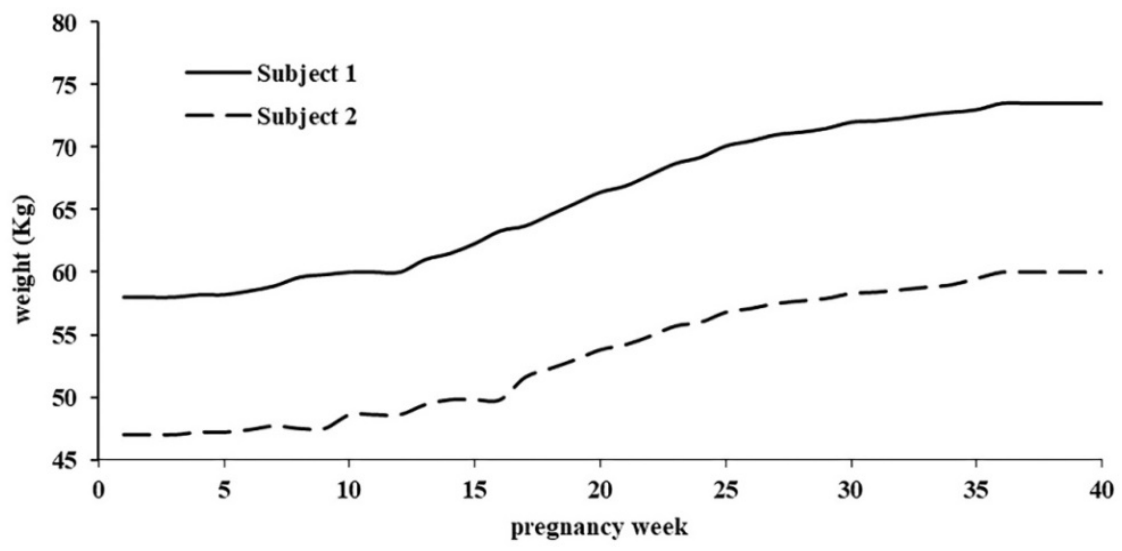

Figure 7. The weight gain graph (2) of the scanned pregnant women (Subjects 1 and 2).

Consequently, the virtual mannequin for the first and third pregnancy period was made based on the weight gain graph proposed in Figure 7 (solid line) as well as body evolution data from the 16th to 26th weeks of pregnancy. Eventually, the virtual mannequin was created for all the maternity period. The result of virtual mannequin estimation using the second approach appeared reasonable as reflected in Figure 8a.

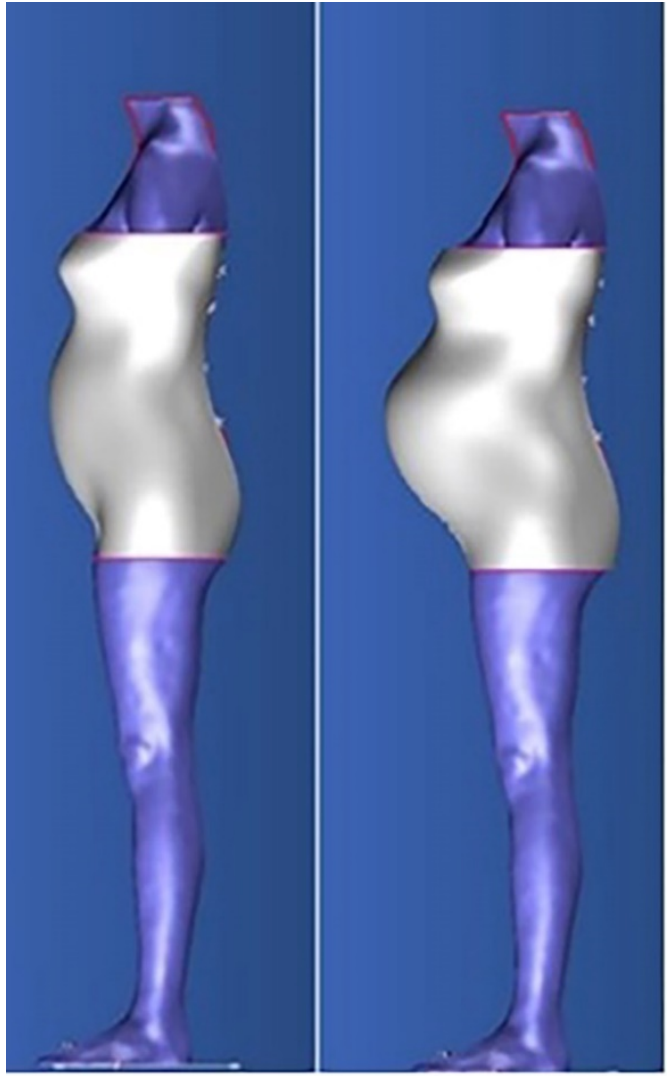

(a)

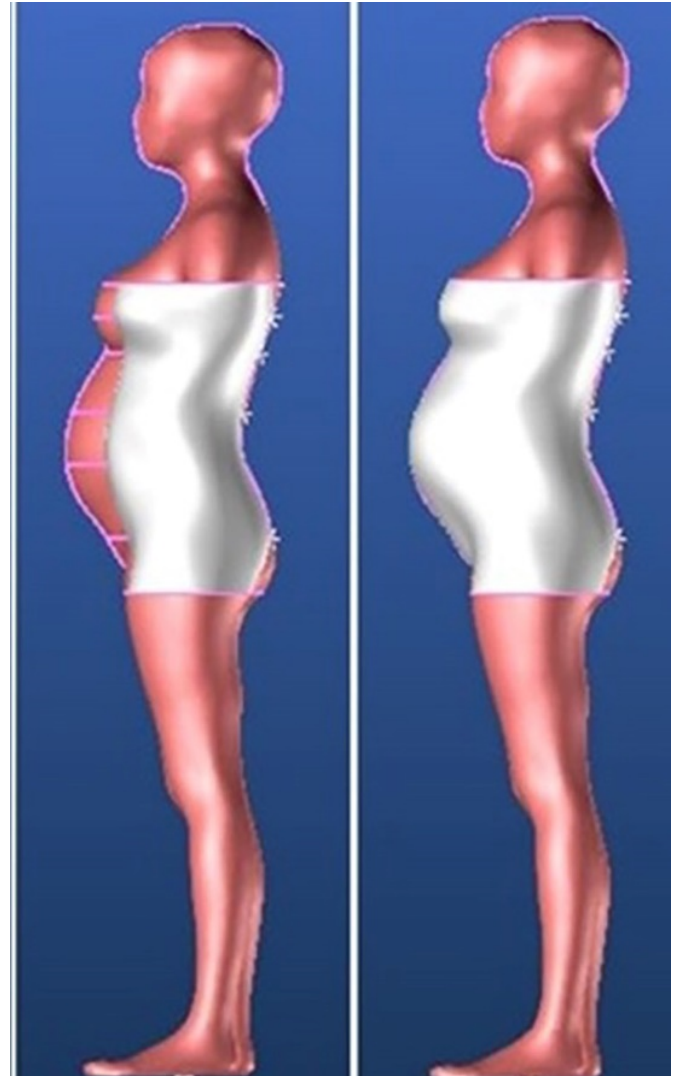

(b)

Figure 8. Developed virtual mannequin for (a) Subject 1 and (b) Subject 2 (20th and 30th weeks of pregnancy based on weight gain graph (2) on the reference bodies. 


\subsubsection{Validation of the Proposed Model}

Scanned data of another pregnant woman (subject 2) was collected and analyzed for the third trimester of pregnancy. The objective was to verify the proposed model of the 3D virtual mannequin for the pregnancy period based on weight. Figure 7 (dashed line) shows the weight gain graphs (2) for subjects 2. It should be noted that body scanning data has been collected during the interval of 30-35th week pregnancy i.e., the third trimester. So, a virtual mannequin for the whole pregnancy period was made regarding weight gain and body evolution of the third trimester of pregnancy for subject 2 .

Consequently, body position adjustment according to legs and spine lines were done for all the bodies through the same process explained for subject 1 . However, the body of the 30th week of pregnancy was considered as a reference body for subject 2 . The reference subject was cut toward its height direction with the same formula represented in Table 1. Also, the other bodies were cut according to their particular spine lines.

The volume value of subject 2 proves an increasing trend for the specific span of time. Nevertheless, the increasing rate is lower than that shown in Figure 5 for subject 1 . It was expected as the analysis was done for a different trimester for each woman. Additionally, it is in agreement with the weight gain graph trend provided in Figure 7.

After that, an adaptive surface of torso was made using the linear upward between the 30th and 35 th weeks of pregnancy for subject 2. Consequently, the virtual mannequin for the whole pregnancy period was created based on weight gain graph (2) and body evolution of subject 2 (Figure $8 b$ ).

\subsubsection{Garment Block Pattern Development}

In the following, a garment block pattern was designed for a pregnant woman (subject 2) using the developed adaptive mannequin. The basic anthropometric feature points of the virtual mannequin (such as shoulder tips, front neck, side waist, side belly, and side hip) were defined as displayed in Figure 9a. These anthropometric feature points help to outline the limits of the pregnant body size. Then, the conical principle developed by Efrat [41] was applied to determine the shape controlling points for breast and scapular of the virtual pregnant mannequin (subject 2). Shoulder blades, the sides of the body and the middle lines for the back and front were used to generate the triangular planes for the front and back of the block pattern as shown in Figure $9 \mathrm{~b}$. Each triangle was formed by connecting two adjacent limit points and the corresponding apex (breast points for the front and scapular points for the back). The projections of the triangles were applied to assemble front and back bodice pattern pieces while the adjustable ease allowance was allocated. It should be mentioned that the shape of the breast was defined with the connection between the curves near the breast area and straight lines distant from the breast.

In addition, a number of angular lines were applied to determine a precise curve for the armpit as represented in Figure 10. The intersection points between the angular lines and the body were made and the armhole curves were formed by connecting the intersection points.

Subsequently, a set of special planes was assigned in the position of waist, belly, hip, and breast, which are important positions for garment design considering the body morphology of a pregnant woman. The distance between the planes and the ground floor (which was considered as reference) was adjusted until the numbers of the planes and distances were appropriate to model the body morphology of the pregnant woman (subject 2). After that, the curves were made on the body while adaptable ease allowance was allocated to each curve [22,42]. 


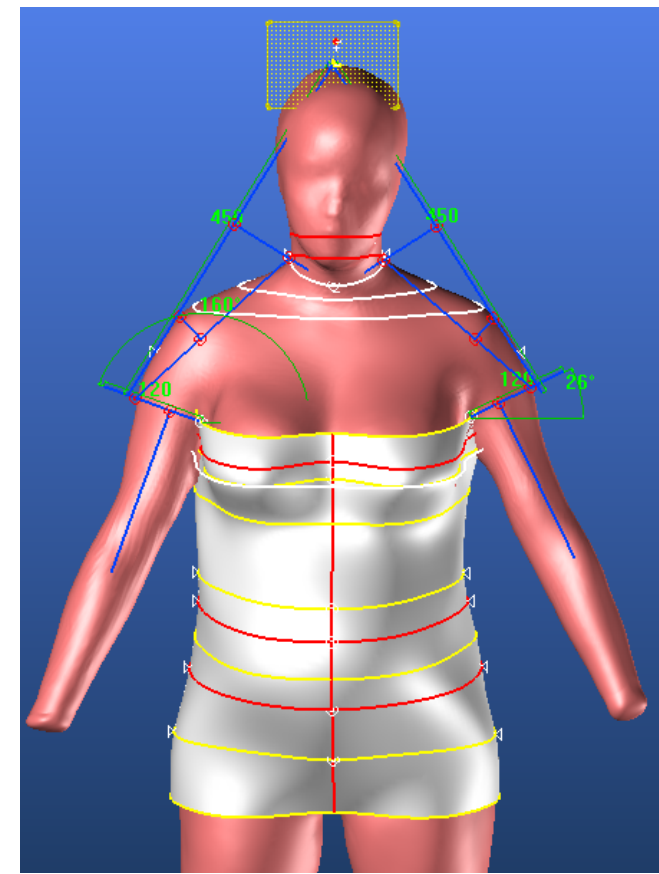

(a)

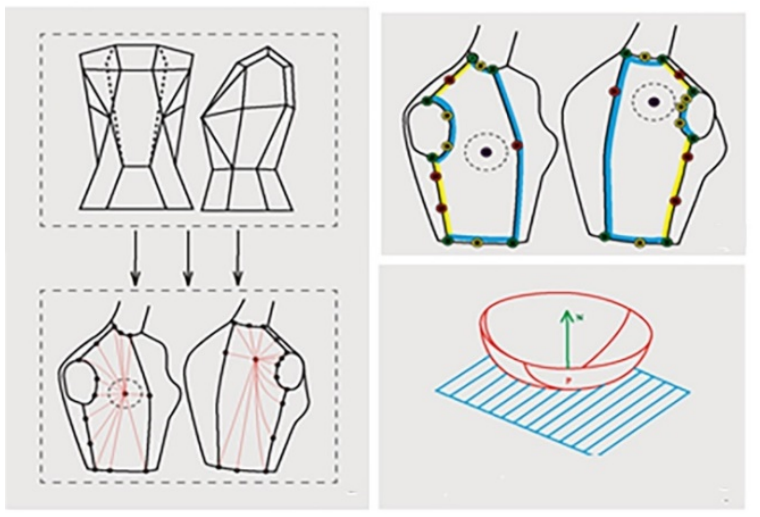

(b)

Figure 9. (a) The anthropometric feature points and (b) general principle of the garment block pattern design [41].

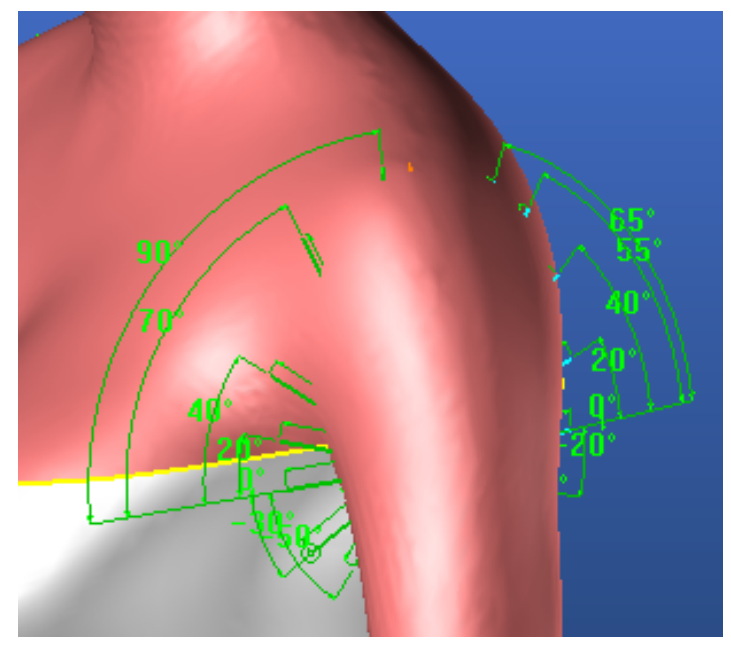

Figure 10. Angular lines for precise armpit curve development.

Finally, the garment block pattern was modeled by triangulating and assembling different parts illustrated in Figure 11a. It should be mentioned that there was no specific requirement for the number and size of triangular meshes as the fabric properties would be given in the following section (virtual try-on).

Then, the flattering operation was applied to obtain the corresponding 2D pattern for the garment block pattern. The garment block pattern consists of eight different pieces in which four are for the front and four are for the back. The flattened 2D pattern was exported into Modaris software for adjustment in order to fulfill the desired properties used in the apparel industry. All the pieces were sewn together in the Modaris environment. 
It should be taken into consideration that the body of a pregnant woman experiences enormous changes only in the torso and as a result, the other parts of the scanned body were merged to develop a complete mannequin for the pregnant woman.

Finally, the garment was tried on the virtual mannequin of the pregnant woman while a number of darts were tested for different pieces of the block pattern. As a result, two armpit darts were placed for the two upper front pieces of the pattern due to the better garment fitting on the body. In addition, an elastic seam was employed to improve the fitting of the garment on the body. It should be noted that there are various types of seams that have been developed to do different jobs. The most important aspect of a well-constructed sewn seam is strength, elasticity, durability, security, and appearance. The elastic seam was applied for the proposed block pattern since the elastic seam enables the material to support its shape of the forces encountered for the end use of the sewn item.

In fact, the available maternity garments on the stock have initially been analyzed to obtain a rough estimation of the suitable seam position for the best appearance and fit. Then, the trial and error method was applied to determine the appropriate position of the seam for the developed block pattern in the present study.

Therefore, the patterns were simulated having an elastic seam for the block pattern. It should be taken into consideration that the pieces were modified in a 2D environment (Modaris software) to obtain a well-fitted pregnancy block pattern and the modified pattern was simulated in a 3D virtual environment. In a few words, the sequence of design-display-evaluation correction was performed repeatedly until a satisfying design was attained.

The final garment block pattern and the corresponding virtual try-on result are represented in Figure 11b-d. As can be seen, the block pattern with armpit darts suggested the appropriate fit on the pregnant virtual mannequin (subject 2). Characteristics of the formerly manufactured fabric were assigned to the design pieces to virtually try on the developed garment block pattern on the body. It should be noted that the thickness of the produced fabric for electromagnetic shielding clothing in our previous work was $3.37 \mathrm{~mm}$ and the areal density was $0.136 \mathrm{~g} / \mathrm{cm}^{2}$ while the woven fabric was made of cotton yarns and hybrid conductive yarns (consist of PA6, 6, carbon black and multiwall carbon nanotube).

\section{Results and Discussion}

\subsection{Three-Dimensional (3D) Virtual Mannequin Generation}

There is a serious lack of mannequins for pregnant women in the apparel and design industry. Hence, it was suggested to develop a 3D virtual mannequin for the pregnancy period for a particular case. Then, the model was generalized for other pregnant women based on weight gain throughout the pregnancy. It should be mentioned that the final purpose of our work was to develop a comfortable garment with electromagnetic shielding effectiveness potential to protect both mother and fetus against radiation while the present study focused on a customized 3D virtual garment design for pregnant women.

For this purpose, the alterations of a pregnant body (subject 1) between the 16th and 26th weeks of maternity period (second trimester) were observed. The body modification of the torso (between the crotch and underbust) was analyzed. The collected data was applied to generate a 3D adaptive virtual mannequin using the linear upward week-to-week for the pregnant woman (subject 1). This created surface for torso was fitted on the virtual scanned body for all the weeks of the second trimester.

Even though the virtual mannequin was created for the second trimester of the pregnancy period, there is still a desperate need for the entire nine months of pregnancy period to apply in the garment design sector. It seems impossible to scan a pregnant body every week without any interruption or gap in between. Several problems might cause gaps since each pregnant woman individually experiences changes in her physical and mental states during pregnancy. For instance, one pregnant woman may be advised to take an absolute rest for a duration or she is not sure the week number of pregnancy 
especially during the first weeks. As a result, the body-scanning data was not available for all the weeks of pregnancy for subject 1 .

Therefore, the possibility of mannequin development for the whole pregnancy period was investigated using the available body scan data for the specified duration of pregnancy. It was proposed to define a correlation between weight gain and changes in the pregnant body. This means that the body evolution for the whole pregnancy period based on available data of body scanning and weight gain was predicted to make a virtual mannequin for whole maternity length.

To that end, a graph of weight gain (graph 2) was used to generate a virtual mannequin for the pregnant woman. The mannequin that was created was well-matched with the real body evolution during the pregnancy period. It means the developed mannequin by weight gain was in good agreement with real body shape during pregnancy.

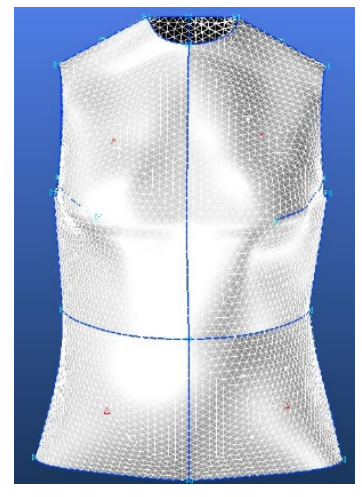

(a)

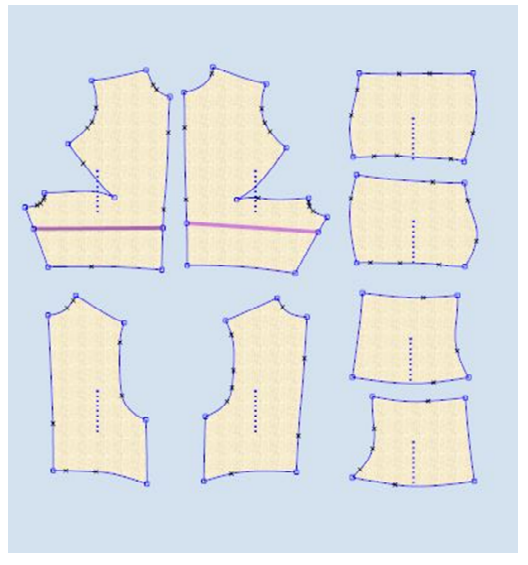

(b)

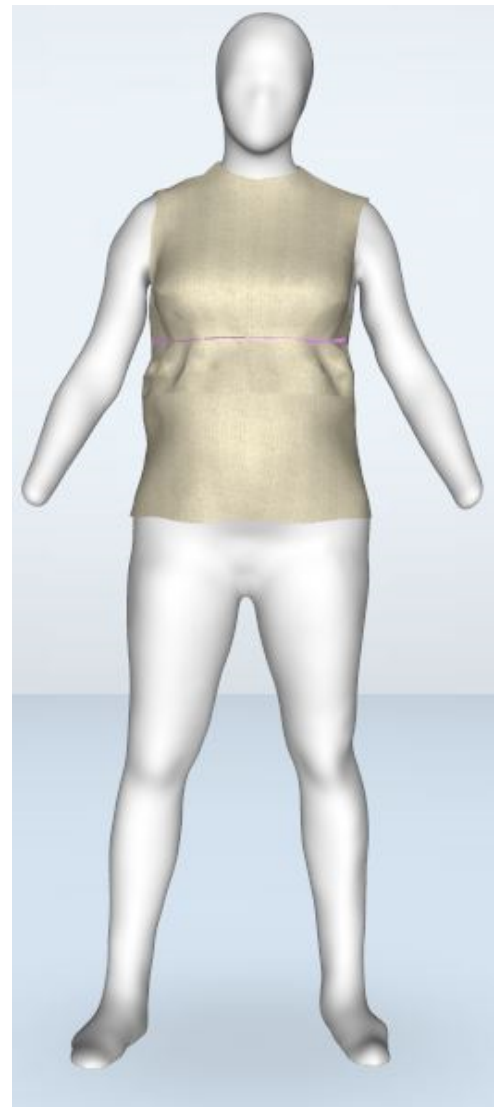

(c)

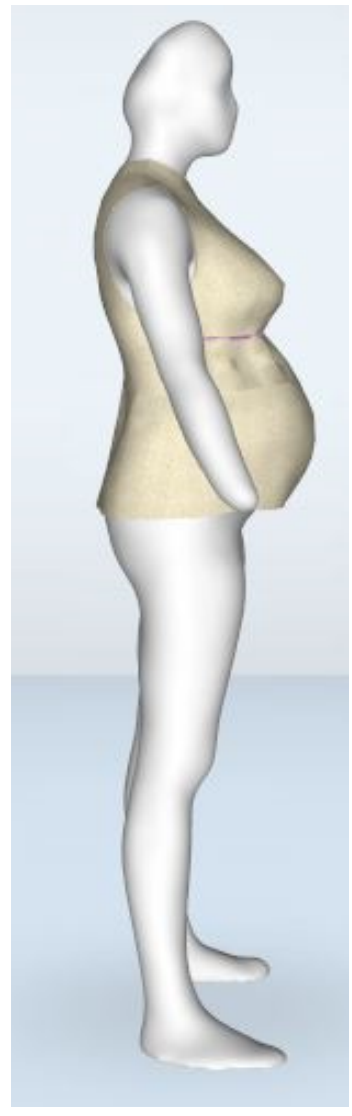

(d)

Figure 11. (a) Front of the garment block pattern in Design Concept environment for (Subject 2), (b) the garment block pattern, (c) front and (d) side view of the corresponding virtual try-on.

\subsection{Validation of the Proposed Model}

Scanned data of another pregnant woman (subject 2) was collected and analyzed for a different period of pregnancy to verify the proposed model of the $3 \mathrm{D}$ virtual mannequin based on weight gain.

Referring to the previous argument in this study, the objective was to create a suitable mannequin for the whole pregnancy period. The expected weight gain of a woman throughout pregnancy along with her initial weight before pregnancy was the main data based on which mannequin was developed. Therefore, the proposed model for mannequin creation based on weight gain had to be confirmed for further investigations on garment design. The body scan data of another pregnant woman was applied to estimate the $3 \mathrm{D}$ virtual mannequin according to her weight gain. The idea was to prove that the 
created mannequin was appropriate. The scan data collected from the third trimester of pregnancy for the second pregnant (subject 2) although it was collected for the second trimester for the first one (subject 1). Finally, the mannequin for the whole pregnancy length was made for the second woman based on her weight gain during pregnancy and the result was promising.

It was suggested that the proposed model to make a 3D virtual mannequin based on weight gain was valid for two different pregnant women. Therefore, 3D virtual mannequins can be generated based on weight gain expectancy for each pregnant woman. Moreover, this model can be applied in the ready-to-wear sector.

\subsection{Garment Block Pattern Development}

A basic garment block pattern was designed in connection with the developed virtual mannequin for a pregnant woman (subject 2). For this purpose, firstly, the virtual mannequin developed for the torso was merged with the scanned body of the pregnant woman (subject 2 ) for the 35th weeks of pregnancy in the Geomagic Design X environment. The reason was correlated to the considerable changes of torso during pregnancy while the evolution of the other parts of the body was negligible. A block pattern was made in the Design Concept environment using the anthropometric feature points and characteristic lines and curves made for the virtual mannequin. The garment block pattern was flattened and the developed garment block pattern for the pregnant woman was virtually tried on the virtual mannequin of the pregnant woman (subject 2). The result demonstrated that the developed block pattern was virtually well-fitted on the developed mannequin of the pregnant woman (subject 2) while the characteristics of the manufactured fabric for the electromagnetic shielding were given to the pattern pieces. Thus, the proposed method can be applied to produce a fitted pregnancy garment for electromagnetic shielding application in our further work.

\section{Conclusions}

This work concentrated on developing a virtual well-fitted block pattern garment for a pregnant woman with the potential of applying in protective garment manufacturing. Therefore, the body morphology evolution was analyzed and the adaptive mannequin surface was generated for the specified period of pregnancy. Then, the virtual surface was verified by available scan bodies. After that, a virtual mannequin was generated for the whole pregnancy period based on the weight gain graph of pregnancy for the woman (subject 1). The reason is correlated to mannequin making for pregnancy period using the available data of the women e.g., the weight and size before pregnancy and the weight gain during pregnancy.

Next, a similar process was applied to make a virtual surface for another scanned pregnant body (subject 2) in order to validate the proposed model. The predicted results were meaningful for the second studied case (subject 2) as well. In fact, the mannequin produced for two different pregnant women regarding their weight gain confirmed the accuracy of the proposed method of mannequin estimation for the whole pregnancy period. The volume expectation was in agreement with actual body evolution throughout pregnancy. As a result, this new approach was successful in mannequin development according to weight gain during maternity.

After that, the developed adaptive mannequin was applied to design a virtual block pattern for the pregnant woman (subject 2). The result of virtual try-on suggested that the garment block pattern was well-fitted on the 3D virtual mannequin while two armpit darts and two elastic seams were assigned to the two-upper front pieces of the pattern. It was also inferred that the developed mannequin based on a woman's weight before pregnancy and her weight gain expectancy during the maternity period is essential to design a comfortable protective garment for pregnant women considering their body deformations. Therefore, in our further work the mannequin and designed garment block pattern developed here will be used to generate a personalized protective garment against electromagnetic waves using the formerly manufactured shielding fabric for different trimesters of pregnancy. 
Author Contributions: Conceptualization, methodology, and software, M.J.T. and P.B.; writing-Original draft preparation, M.J.T., and P.B.; writing-Review and editing, M.J.T., P.B., I.C., A.C., C.C., C.L., Y.C. and L.W.; supervision and funding acquisition, C.C., C.L., Y.C.

Funding: This work has been comprehended in the framework of Erasmus Mundus Joint Doctorate Project-SMDTex (Sustainable Management and Design for Textiles), which is financed by the European Commission (Grant no.: n²016-1353/001-001-EMJD).

Acknowledgments: The authors would like to thank Mr. Francois Dassonville for his help in Geomagic Design X software work.

Conflicts of Interest: The authors declare no conflict of interest.

\section{References}

1. Toghchi, M.J.; Campagne, C.; Cayla, A.; Bruniaux, P.; Loghin, C.; Cristian, I.; Burgnies, L.; Chen, Y. Electrical Conductivity Enhancement of Hybrid PA6,6 Composite Containing Multiwall Carbon Nanotube and Carbon Black for Shielding Effectiveness Application in textiles. Synth. Met. 2019, 251, 75-84. [CrossRef]

2. Pheasant, S.; Haslegrave, C.M. Bodyspace: Anthropometry, Ergonomics, and Design of Work; CRC Press: Boca Raton, FL, USA, 2006.

3. Wang, C.C.L.; Wang, Y.; Yuen, M.M.F. Design automation for customized apparel products. CAD Comput. Aided Des. 2005, 37, 675-691. [CrossRef]

4. Nkambule, M.T. Apparel Sizing and Fit Preferences and Problems of Plus-Size. Swazi Working Women. Ph.D. Thesis, University of Pretoria, Pretoria, South Africa, 2010.

5. Wang, C.C.L.; Yuen, M.M.F. Editorial: CAD methods in garment design. CAD Comput. Aided Des. 2005, 37, 583-584. [CrossRef]

6. Cichocka, A.; Bruniaux, P.; Frydrych, I. 3D Garment Modelling-Creation of a virtual mannequin of the human body. Fibres Text. East. Eur. 2014, 22, 123-131.

7. McCartney, J.; Hinds, B.K.; Seow, B.L.; Gong, D. Dedicated 3D CAD for garment modelling. J. Mater. Process. Technol. 2000, 107, 31-36. [CrossRef]

8. Wang, J.; Lu, G.; Li, W.; Chen, L.; Sakaguti, Y. Computer-Aided Design Interactive 3D garment design with constrained contour curves and style curves. Comput. Des. 2009, 41, 614-625.

9. Duan, L.; Yueqi, Z.; Ge, W.; Pengpeng, H. Automatic three-dimensional-scanned garment fitting based on virtual tailoring and geometric sewing. J. Eng. Fibers Fabr. 2019, 14. [CrossRef]

10. Liu, Y.; Zhang, D.; Yuen, M.M. Computers in Industry A survey on CAD methods in 3D garment design. Comput. Ind. 2010, 61, 576-593. [CrossRef]

11. LaBat, K.L.; Ryan, K.S. Human Body: A Wearable Product Designer's Guide, 1st ed.; CRC Press: Boca Raton, FL, USA, 2019.

12. Li, J.; Wang, Y. Automatically Constructing Skeletons and Parametric Structures for Polygonal Human Bodies; Digital. Interactive Media Lab, Institute of Automation, Academy of Chinese Sciences: Beijing, China, 2007.

13. Wang, C.C.L. Parameterization and parametric design of mannequins. CAD Comput. Aided Des. 2005, 37, 83-98. [CrossRef]

14. Allen, B.; Curless, B.; Popović, Z. The space of human body shapes. ACM Trans. Graph. 2003, 22, 587. [CrossRef]

15. Abtew, M.A.; Bruniaux, P.; Boussu, F.; Loghin, C.; Cristian, I.; Chen, Y.; Wang, L. Female seamless soft body armor pattern design system with innovative reverse engineering approaches. Int. J. Adv. Manuf. Technol. 2018, 98, 2271-2285. [CrossRef]

16. Hamad, M.; Thomassey, S.; Bruniaux, P. A new sizing system based on 3D shape descriptor for morphology clustering. Comput. Ind. Eng. 2017, 113, 683-692. [CrossRef]

17. Hong, Y.; Zeng, X.; Bruniaux, P.; Liu, K. Interactive virtual try-on based three-dimensional garment block. Text. Res. J. 2017, 87, 1261-1274. [CrossRef]

18. Rudolf, A.; Cupar, A.; Kozar, T.; Stjepanović, Z. Study Regarding the Virtual Prototyping of Garments for Paraplegics. Fibers Polym. 2015, 16, 1177-1192. [CrossRef]

19. Cupar, A.; Rudolf, A. Construction of adapted garments for people with scoliosis using virtual prototyping and CASP method. Ind. Text. 2016, 67, 141-149. 
20. Furferi, R.; Mussi, E.; Servi, M.; Uccheddu, F.; Volpe, Y.; Facchini, F. 3D Acquisition of the Ear Anatomy: A Low-Cost Set up Suitable for the Clinical Practice. In XV Mediterranean Conference on Medical and Biological Engineering and Computing-MEDICON 2019, Proceedings of the Mediterranean Conference on Medical and Biological Engineering and Computing, Coimbra, Portugal, 26-28 September 2019; Magjarevic, R., Henriques, J., Neves, N., de Carvalho, P., Eds.; Springer Nature Switzerland AG: Cham, Switzerland, 2019; Volume 76, pp. 669-678.

21. Uccheddu, F.; Ghionzoli, M.; Volpe, Y.; Servi, M.; Furferi, R.; Governi, L.; Facchini, F.; Piccolo, R.L.; McGreevy, K.S.; Martin, A.; et al. A Novel Objective Approach to the External Measurement of Pectus Excavatum Severity By Means of an Optical Device. Ann. Thorocic Surg. 2018, 106, 221-227. [CrossRef] [PubMed]

22. Thomassey, S.; Bruniaux, P. A template of ease allowance for garments based on a 3D reverse methodology. Int. J. Ind. Ergon. 2013, 43, 406-416. [CrossRef]

23. Cordier, F.; Seo, H.; Magnenat-Thalmann, N. Made-to-measure technologies for an online clothing store. IEEE Comput. Graph. Appl. 2003, 23, 38-48. [CrossRef]

24. Groß, C.; Fuhrmann, A.; Luckas, V. Automatic pre-positioning of virtual clothing. In Proceedings of the 19th Spring Conference on Computer Graphics, New York, NY, USA, 24-26 April 2003.

25. Ho, S.S.M.; Yu, W.W.M.; Lao, T.T.; Chow, D.H.K.; Chung, J.W.Y.; Li, Y. Garment needs of pregnant women based on content analysis of in-depth interviews. J. Clin. Nurs. 2009, 18, 2426-2435. [CrossRef]

26. Lederman, S.A.; Paxton, A.; Heymsfield, S.B.; Wang, J.; Thornton, J.; Pierson, R.N., Jr. Body fat and water changes during pregnancy in women with different body weight and weight gain. Obstet. Gynecol. 1997, 90, 483-488. [CrossRef]

27. Calvo, E.B.; López, L.B.; Balmaceda, Y.D.V.; Poy, M.S.; González, C.; Quintana, L.; Cámera, K.; Barrientos, E.; Berlingueres, S.; Garciarena, S. Reference charts for weight gain and body mass index during pregnancy obtained from a healthy cohort. J. Matern. Neonatal Med. 2009, 22, 36-42. [CrossRef] [PubMed]

28. Georgeta, P.; Alexandra, M.; Sabina, O. Innovative clothing design for women during pregnancy. Ann. Univ. Oradea Fascicle Text. Innov. 2015, 16, 67-72.

29. Rodriguez, C.Q.; Anisimova, A.; Ryan, S.; Troynikov, O. Critical design aspects of maternity support-garments and its contemporary perspective. KnE Eng. 2017, 2, 91. [CrossRef]

30. Sohn, M.; Bye, E. Visual analysis of body shape changes during pregnancy. Int. J. Fash. Des. Technol. Educ. 2012, 5, 117-128. [CrossRef]

31. Wu, G.; Liu, S.; Wu, X.; Ding, X. International Journal of Industrial Ergonomics Research on lower body shape of late pregnant women in Shanghai area of China. Int. J. Ind. Ergon. 2015, 46, 69-75. [CrossRef]

32. FTTS-FA-003. Test Method of Specified Requirements of Electromagnetic Shielding Textiles. In Committee for Conformity Assessment on Accreditation and Certification of Functional and Technical Textiles; TTRI: Taipei, Taiwan, 2003.

33. Zamanian, A.; Hardiman, C. Electromagnetic radiation and human health: A review of sources and effects. EMR Hum. Heal. 2005, 16, 16-26.

34. Hossmann, K.A.; Hermann, D.M. Effects of electromagnetic radiation of mobile phones on the central nervous system. Bioelectromagnetics 2003, 24, 49-62. [CrossRef]

35. Erogul, O.; Oztas, E.; Yildirim, I.; Kir, T.; Aydur, E.; Komesli, G.; Irkilata, C.H.; Irmak, K.M.; Peker, F.A. Effects of electromagnetic radiation from a cellular phone on human sperm motility: An in vitro study. Arch. Med. Res. 2006, 37, 840-843. [CrossRef]

36. Sohlstr, A.; Forsum, E. Changes in adipose tissue volume and distribution during reproduction in Swedish women as assessed by magnetic resonance imaging13. Am. J. Clin. Nutr. 1995, 61, 287-295. [CrossRef]

37. de Onis, M.; Garza, C.; Onyango, A.W.; Borghi, E. Comparison of the WHO Child Growth Standards and the CDC 2000 Growth Charts 1. J. Nutr. 2007, 137, 144-148. [CrossRef]

38. Ölin, A.; Rössner, S. Factors Related to Body Weight Changes During and After Pregnancy: The Stockholm Pregnancy and Weight Development Study. Obes. Res. 1996, 4, 271-276. [CrossRef] [PubMed]

39. Brenner, W.E.; Edelman, D.A.; Hendricks, C.H. A standard of fetal growth for the united states of America. Am. J. Obstet. Gynecol. 1976, 126, 555-564. [CrossRef]

40. Barbara, A.F.; Laros, R.K., Jr. Prepregnancy weight, weight gain, and birth weight. Am. J. Obstet. Gynecol. 1986, 154, 503-509. 
41. Efrat, S. The Development of a Method for Generating Patterns for Garments that Conform to the Shape of the Human Body. Ph.D. Thesis, De Montfort University, Leicester, UK, 1982.

42. Chen, Y.; Zeng, X.; Happiette, M.; Bruniaux, P.; Ng, R.; Yu, W. A new method of ease allowance generation for personalization of garment design. Int. J. Cloth. Sci. Technol. 2008, 20, 161-173. [CrossRef]

(C) 2019 by the authors. Licensee MDPI, Basel, Switzerland. This article is an open access article distributed under the terms and conditions of the Creative Commons Attribution (CC BY) license (http://creativecommons.org/licenses/by/4.0/). 